\title{
Rasgos de personalidad e inteligencia emocional del personal de seguridad pública de Huancayo al 2015
}

\author{
Personality traits and emotional intelligence of the staff of public \\ security of Huancayo to 2015
}

\author{
Félix Rodríguez Isidro ${ }^{\mathrm{a}, *}$ \\ a Universidad Continental, Carrera Profesional de Psicología, Huancayo, Perú
}

\section{RESUMEN}

El objetivo de la investigación fue determinar la relación existente entre los rasgos de personalidad y la inteligencia emocional en el personal de serenazgo y policía municipal integrantes del personal de seguridad ciudadana de la ciudad de Huancayo, asimismo las características que favorecen y refuerzan los rasgos de personalidad que forman parte de la inteligencia emocional, siendo esta la habilidad imprescindible para que las personas adviertan sus estados emocionales, gracias a la comprensión y el control de sus respuestas afectivas frente a situaciones diversas. La investigación es básica, de alcance descriptivo, diseño correlacional. La población fue constituida por el personal de serenazgo y la policía municipal, la muestra fue de 114 miembros, escogidos de forma probabilística. Los instrumentos aplicados fueron, el Inventario Clínico Multiaxial de Millón II para los rasgos de personalidad, y el inventario de Inteligencia Emocional de Baron para manejo emocional. Entre los resultados, se tiene que los rasgos de personalidad en los miembros de serenazgo y policía municipal es el dependiente 23 $\%$, narcisista $20 \%$, compulsivos $19 \%$, agresivo sádico $18 \%$, esquizoide $6 \%$, evitativo $4 \%$, histriónico $3 \%$, pasivo agresivo $2 \%$, y autoderrotista $2 \%$; respecto al manejo emocional tiene un buen autoconcepto $53 \%$, mantiene buenas relaciones interpersonales $63 \%$, adecuada adaptabilidad $93 \%$, controlan sus impulsos $55 \%$, son felices $76 \%$. En conclusión, los rasgos de personalidad guardan relación directa y positiva con la inteligencia emocional.

Palabras clave: Inteligencia emocional, rasgos de personalidad, seguridad ciudadana.

\begin{abstract}
The aim of the research was to determine the relationship between personality traits and emotional intelligence in the staff of local security (serenazgo) and municipal police, members of the security personnel staff in Huancayo city, also features that support and reinforce the personality traits which are part of emotional intelligence, this ability being essential for people to warn their emotional states, in order to understanding and control their emotional responses to various situations. This is a basic research in a descriptive scope and correlational designed. The sample population was constituted by the serenazgo staff and the municipal police, with a sample of 114 members, chosen in a probabilistic way. Applied instruments were Million Clinical Multiaxial Inventory II for personality traits and Baron Emotional Intelligence Inventory for emotional management. Results obtaining show that personality traits in members of serenazgo staff and municipal police is: dependent in $23 \%$, narcissistic in $20 \%$, compulsive in $19 \%$, aggressive sadistic in 18 $\%$, schizoid in $6 \%$, avoidant in $4 \%$, histrionic in $3 \%$, passive aggressive in $2 \%$, and self-defeatist in $2 \%$. About handling emotions has a good self-concept in $53 \%$, maintain good interpersonal relationships in $63 \%$, adequate adaptability in $93 \%$, control their impulses in $55 \%$ and are happy in $76 \%$. Concluding with personality traits keep a direct and positive relationship with emotional intelligence.
\end{abstract}

Keywords: Emotional intelligence, personality features, city safety.

Historial del artículo:

Recibido, 26 de noviembre de 2015; aceptado, 19 de febrero de 2016; disponible en línea, 30 de marzo de 2016

* Magister en educación, Doctor en psicología, Docente de la Universidad Continental.

Correo: frodriguez@continental.edu.pe 


\section{INTRODUCCIÓN}

Hoy en día la labor que cumplen muchas personas dentro de la sociedad trae consigo una serie de dificultades a nivel emocional y muchas veces puede deberse a los rasgos de personalidad que tienen dichas personas. Es así que los trabajadores que tienen a cargo el control de bienestar social tal como la policía municipal y serenazgo deben tener mayor control de impulsos, debido a que mantener el orden de nuestra ciudad implica estar sometido a situaciones de estrés, por lo que la inteligencia emocional debe ser primordial para el buen desarrollo del trabajo de estas personas Bisquera (1). Es por ello que se realiza la presente investigación, para dar a conocer cuál es la relación entre los rasgos de personalidad y la inteligencia emocional.

Una de las variables a investigar dentro del presente inteligencia emocional en el personal de serenazgo y policía municipal de la ciudad de Huancayo. Justificamos esto ya que la inteligencia emocional es un aspecto muy importante en el desarrollo de nuestras vidas, aspectos que pueden ser determinantes al momento de la interacción con los demás Bisquera 4), asimismo, lo son para poder obtener éxito tanto en el área laboral, familiar, profesional y social, también influye los rasgos de personalidad que cada persona tiene, la inteligencia emocional es un área que puede ser educada Bisquera (5), el resultado de esta educación depende de los rasgos de personalidad y el carácter Cloninger (6), además es moldeable el resultado de esta educación, y ayudaría a las personas a un desarrollo favorable en todos los ámbito de su vida.

A su vez Matassini (7) menciona que, si existe una correlación positiva entre ambas variables,

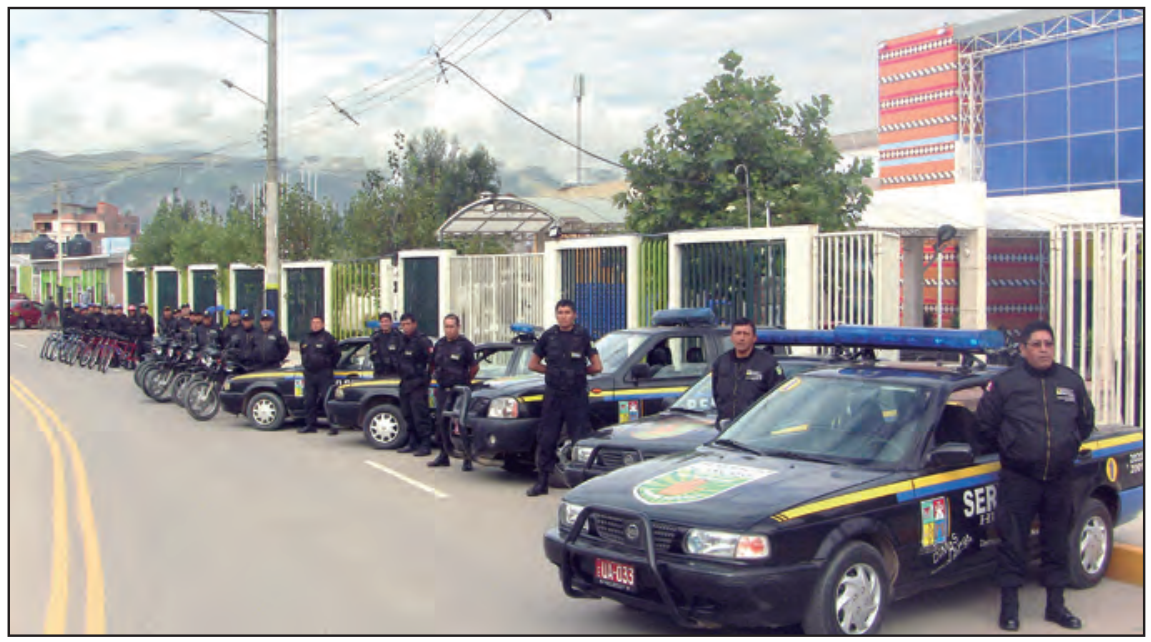

Figura $N^{\circ}$ 1: Personal de Serenazgo de Huancayo, Perú.

Fuente: Municipalidad Provincial de Huancayo

trabajo es la inteligencia emocional que es una habilidad necesaria para conocer, comprender y controlar sus respuestas emocionales de acuerdo a las situaciones que enfrente (2). Siendo la raíz de este control las emociones que son las que producen una activación que nos proporciona la energía necesaria para responder, rápidamente, a un estímulo que atente a nuestro bienestar físico, psicológico, sin embargo, las emociones pueden influir al tomar las malas decisiones por no saberlas controlar.

Otra de las variables son los rasgos de personalidad que son características psíquicas de una persona que determina que los individuos actúen de manera diferente ante una determinada circunstancia Boeree (3).

Nuestra hipótesis muestra que existe correlación directa y positiva entre los rasgos de personalidad e encontrándose que a un mayor nivel de inteligencia emocional le corresponde un mejor clima organizacional, también que existe una relación directa y positiva entre la dimensión adaptabilidad de la inteligencia emocional y el variable clima organizacional.

Reafirmando entonces que a mayor inteligencia emocional mejores resultados en el desempeño laboral dentro de las organizaciones y mayor tendencia al éxito tanto, personal, profesional y familiar.

Por lo planteado anteriormente, la relación entre los rasgos de personalidad y la inteligencia emocional en el personal resalta que el desarrollo de las aptitudes emocionales permite desarrollar la capacidad de manejar las emociones idóneas para cada acción y regular su manifestación, manteniendo el equilibrio emocional; transmitiendo estados de ánimo para 
generar actitudes y respuestas positivas; aprendiendo a evaluar el costo emocional de situaciones y acciones; desarrollando destrezas sociales, forjando y manejando relaciones con sus allegados, Blasco (8). De esta forma, las emociones pueden guiar todas las actitudes de nuestra vida hacia pensamientos y hábitos constructivos, que mejoren en forma absoluta los resultados finales que queremos alcanzar.

La importancia radica en que se debe desarrollar la inteligencia emocional, para que junto a los rasgos de personalidad permitan dar diferentes respuestas ante diversas situaciones que en su mayoría son de violencia por el tipo de trabajo que tiene el personal de la policía municipal y serenazgo.

\section{MATERIAL Y MÉTODOS}

El diseño fue descriptivo correlacional, Hernandez (9), permitiéndonos identificar y conocer la naturaleza de una situación en la medida en que ella existe durante el tiempo de estudio; por tanto no hay un tratamiento específico. Este estudio tiene como propósito evaluar la relación existente entre la inteligencia emocional y los rasgos de personalidad.

La población estuvo constituida por el personal de serenazgo y policía municipal de la ciudad de Huancayo, con una muestra probabilística de 114 serenos y policías municipales. La selección se realizó mediante muestreo aleatorio simple, Arias (10), tomando como marco muestral el listado de serenos y policías municipales que laboran en la Municipalidad de Huancayo, lo que permitió identificar al personal seleccionado.

Para esta investigación se aplicó la técnica de Psicometría siendo su instrumento un cuestionario de personalidad, Inventario Clínico Multiaxial de Millón (11) y el cuestionario de inteligencia emocional,

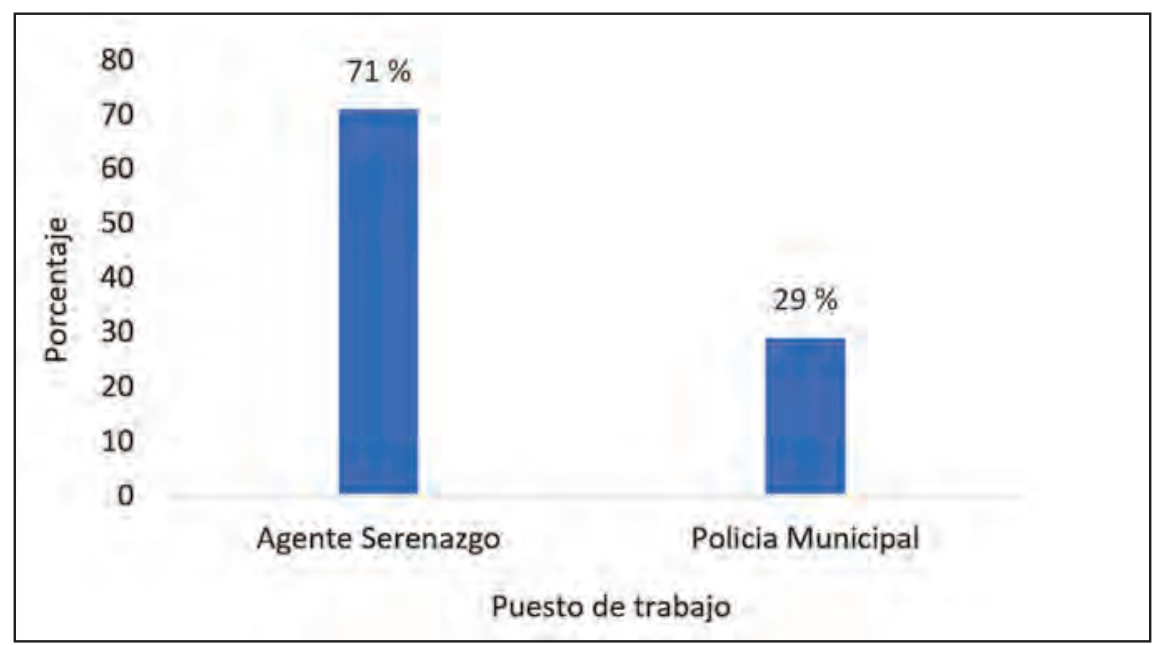

Figura $\mathrm{N}^{\circ} 1$ : Distribución de la muestra por puesto de trabajo.

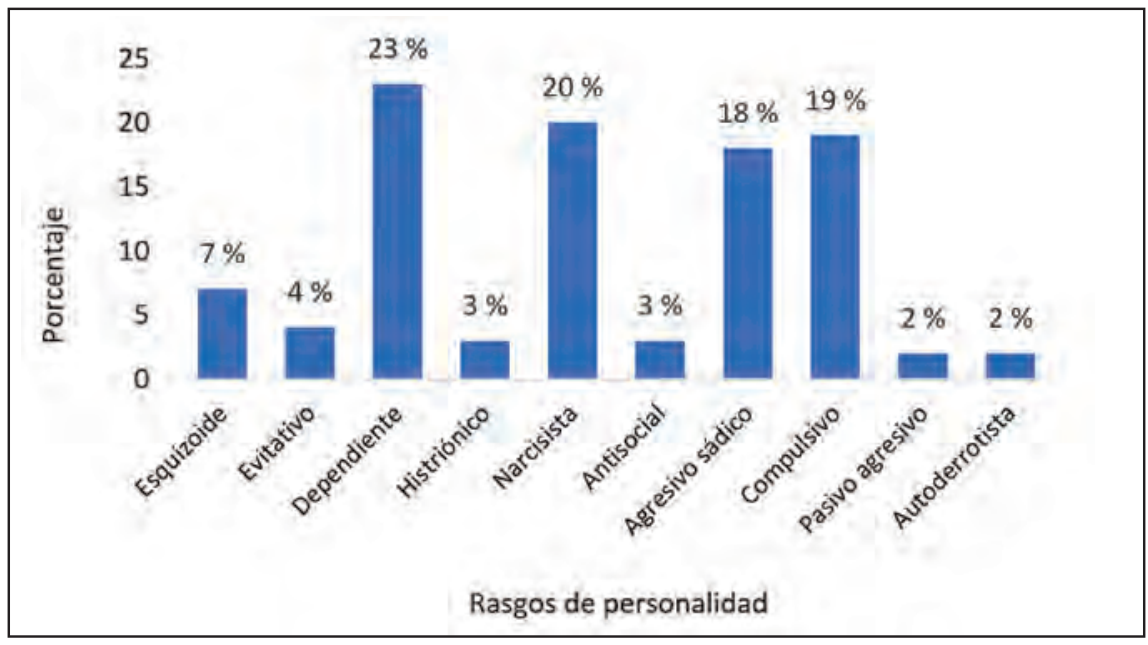

Figura $\mathrm{N}^{\circ}$ 2: Distribución de la muestra por rasgos de personalidad. 
Inventario Emocional Baron (12), para personal de serenazgo y policía municipal de la ciudad de Huancayo. Para el procesamiento de datos se utilizó el software del test de Baron (I-CE) y el programa SPSS 22 para el análisis de los datos.

\section{RESULTADOS}

De la muestra el $71 \%$ son Agentes de Serenazgo y el 29 \% son de la Policía Municipal (figura 1).

Los rasgos de personalidad en los miembros de Serenazgo y Policía Municipal es el dependiente $23 \%$, narcisista $20 \%$, compulsivos $19 \%$, agresivo sádico 18 $\%$, esquizoide $6 \%$, evitativo $4 \%$, histriónico $3 \%$, pasivo agresivo $2 \%$, y autoderrotista $2 \%$ (figura 2 ).
En el área intrapersonal los serenos y policías municipales el $53 \%$ tiene un buen autoconcepto, el $44 \%$ se siente autorrealizado, el $2 \%$ se siente independiente y $1 \%$ tiene conocimiento emocional de sí mismo (figura 3).

En el aspecto inter personal el $63 \%$ de los miembros del serenazgo y policía municipal, mantiene buenas relaciones interpersonales, el $36 \%$ tiene responsabilidad social y el $1 \%$ empatía (figura 4 ).

En el área de adaptabilidad el 92,04 \% de los serenos y policías municipales se adapta a la realidad, el 4,425 $\%$ tiene un buen manejo de solución de problemas y el $3,54 \%$ una buena flexibilidad (figura 5).

Los serenos y policías municipales el en área de tensión, el 55,75 \% controla sus impulsos y 44,25\% tiene buen manejo de la tolerancia al estrés (figura 6).

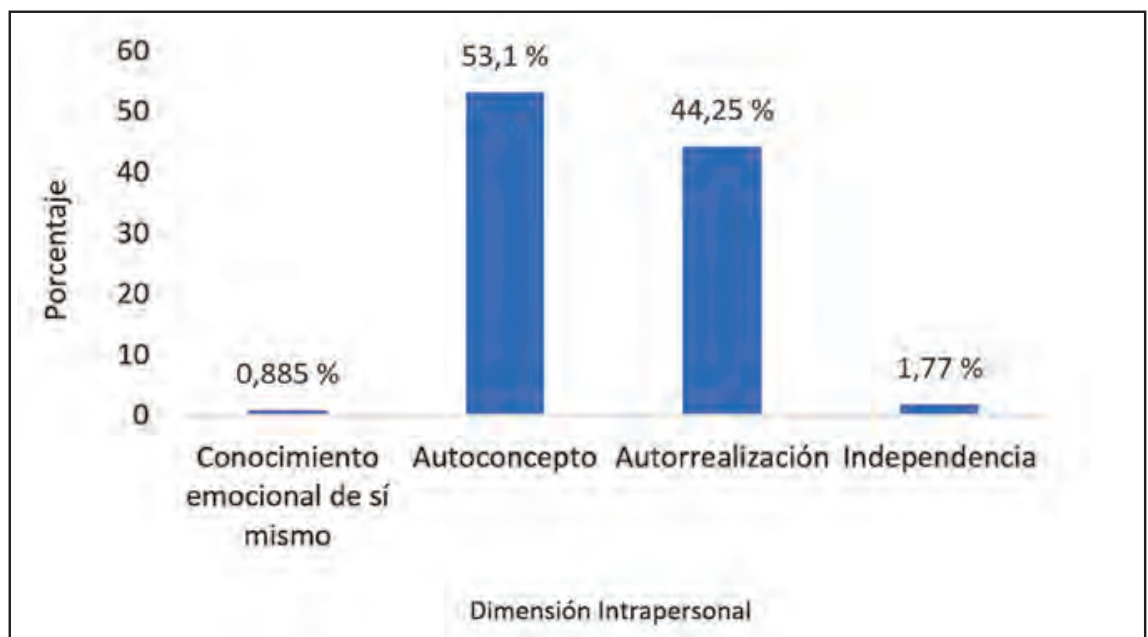

Figura $\mathrm{N}^{\circ}$ 3: Distribución de la muestra por desarrollo de inteligencia emocional (dimensión intrapersonal).

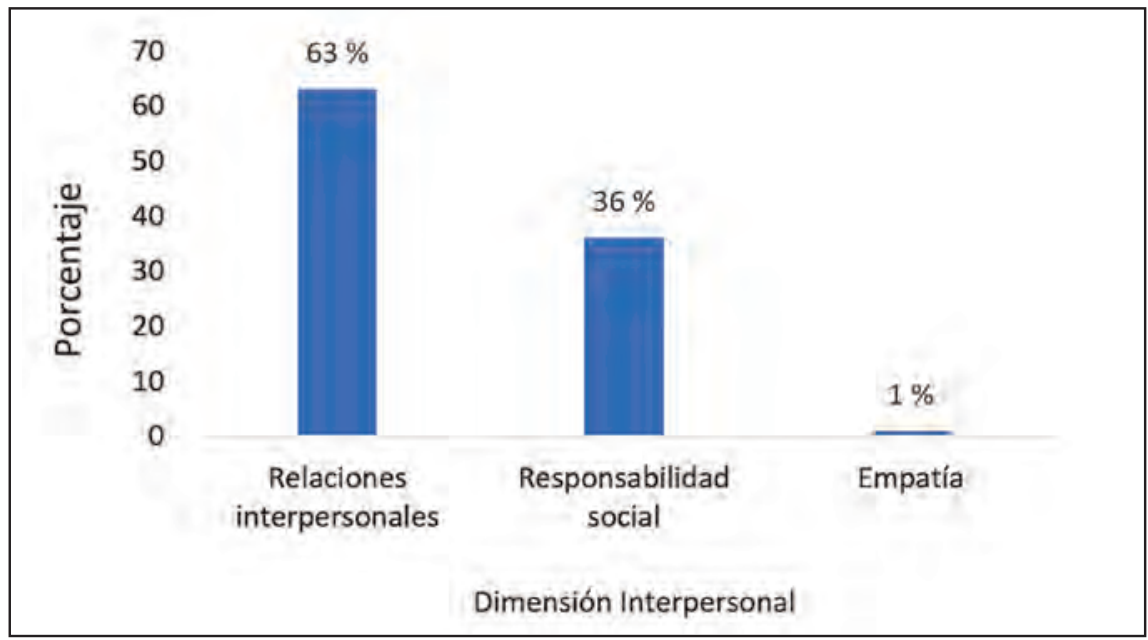

Figura $\mathrm{N}^{\circ} 4$ : Distribución de la muestra por desarrollo de inteligencia emocional (dimensión Interpersonal). 


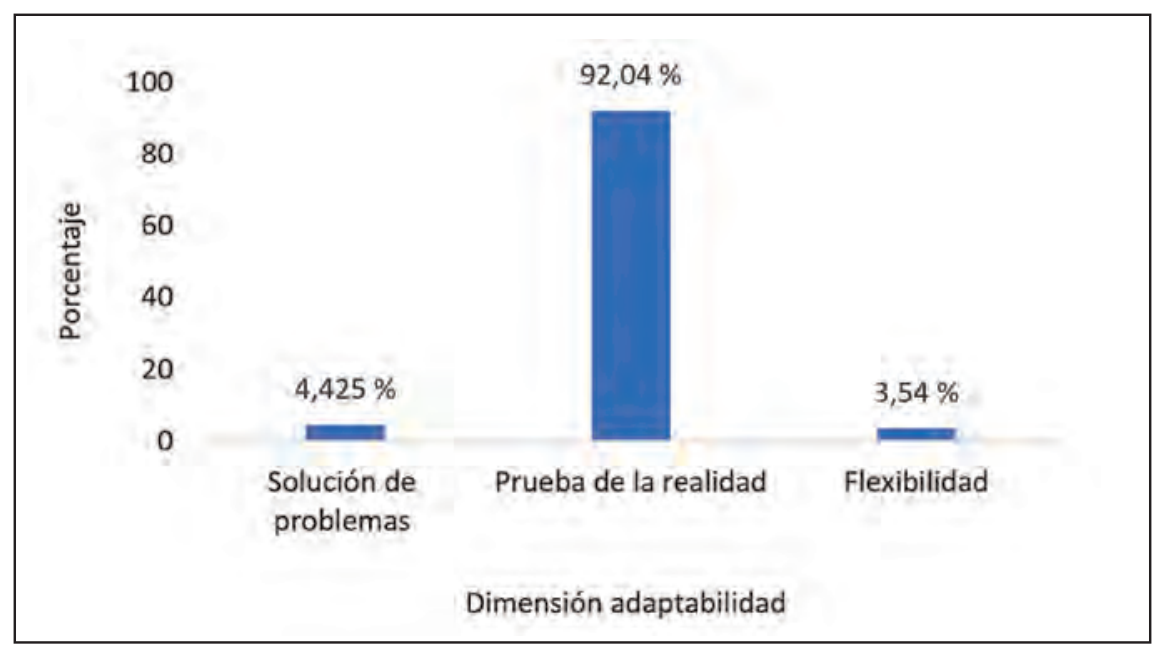

Figura $N^{\circ}$ 5: Distribución de la muestra por desarrollo de inteligencia emocional (dimensión adaptabilidad).

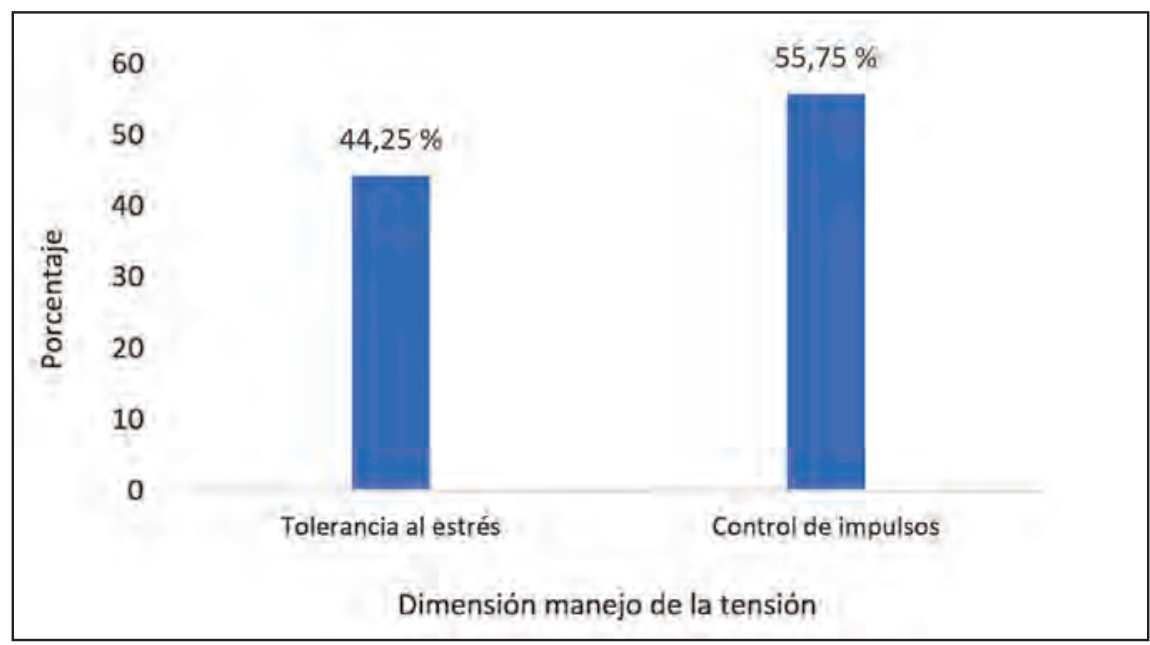

Figura $\mathrm{N}^{\circ}$ 6: Distribución de la muestra por desarrollo de inteligencia emocional (dimensión manejo de tensión).

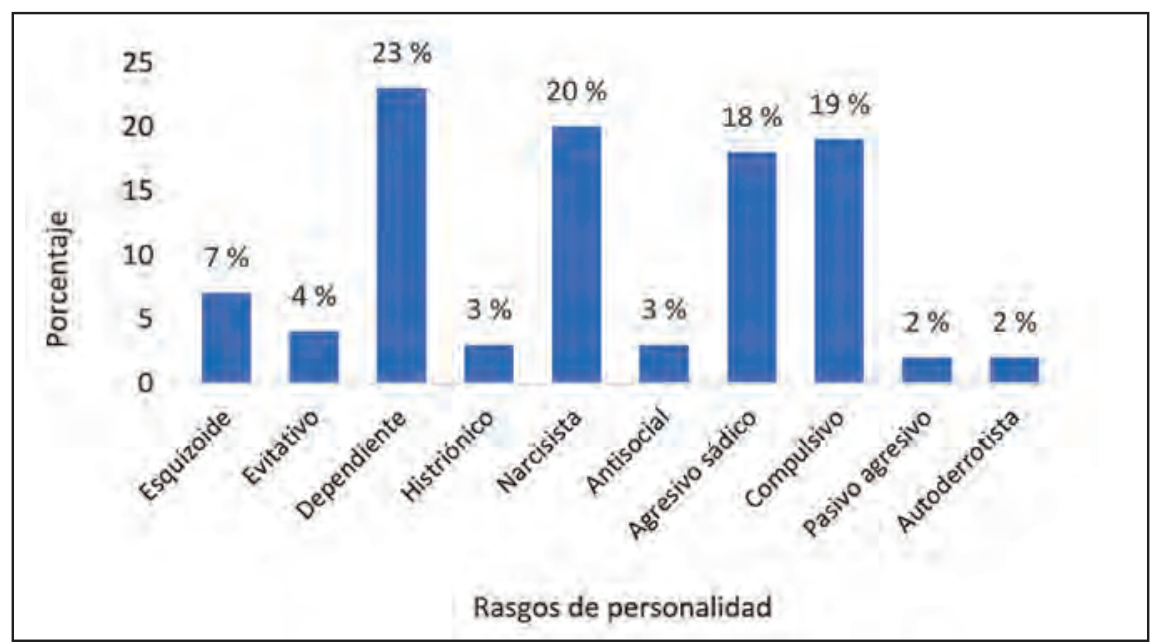

Figura $\mathrm{N}^{\circ}$ 7: Distribución de la muestra por desarrollo de inteligencia emocional (dimensión estado de ánimo en general). 
La figura 7 muestra que por lo general los serenos y policías son felices en un $76 \%$ y el $24 \%$ son optimistas.

\section{DISCUSIÓN}

Respecto a la relación entre los rasgos de personalidad y el área interpersonal de la inteligencia emocional, se encontró que el rasgo de personalidad dependiente tiene buenas relaciones personales, responsabilidad social, y se asemejan a los rasgos de personalidad que se manifiesta a través de la preocupación por los demás en exceso, Montaño (13) que viven su vida a través de los demás y para los demás, tiene que adoptar un papel pasivo en sus relaciones interpersonales, el narcisista también muestra un alto nivel de relaciones interpersonales, ya que necesita que los demás admiren su grandeza, poniendo de lado su responsabilidad social y una escasa o nula empatía hacia los demás; el rasgo compulsivo a su vez refleja también buenas relaciones interpersonales ya que necesita que los demás admiren su perfecto trabajo, compartiendo una escasa responsabilidad social y un pequeño porcentaje de empatía, por su parte el agresivo sádico tiene un nivel medio de relaciones interpersonales, baja responsabilidad social y baja empatía, en cuanto al histriónico muestra buenas relaciones personales, por el mismo hecho que ellos necesitan ser admirados por los demás, por otro lado el esquizoide, evitativo, antisocial, pasivo agresivo y autoderrotista muestran escasa relación interpersonal por sus mismos rasgos de personalidad, poca responsabilidad social y escasa empatía.

En los resultados se encontró que los serenos y policías municipales con rasgos de personalidad dependiente tienen un buen manejo de solución de problemas, reconocen la realidad y un pequeño porcentaje de flexibilidad, en cuanto al narcisista, tiene un mejor desarrollo de reconocer la realidad en la que se encuentran y adaptarse a esta, lo mismo que el compulsivo y el agresivo sádico, por su parte, el esquizoide, evitativo, antisocial, histriónico, pasivo agresivo y autoderrotista, muestran una escasa solución de problemas, que les cuesta adaptarse a la realidad en la que viven y una escasa flexibilidad.

Asimismo, en los resultados se muestra la relación de los rasgos de personalidad y el área de estado general de la inteligencia emocional Contreras (14), donde se puede ver que tanto el dependiente, narcisista, agresivo - sádico y compulsivo la mayor parte del tiempo su ánimo general es de felicidad y optimismo, al contrario del esquizoide, evitativo, antisocial histriónico, pasivo agresivo y autoderrotista, que muestran en general de poca felicidad y optimismo.

Por los resultados obtenidos y la contrastación con el marco teórico en referencia Goleman (2). A través del manejo emocional un personal de serenazgo y policía municipal es eficiente y eficaz en sus intervenciones con el objetivo de lograr la conciliación y resolución de problemas, con dialogo asertivo con la finalidad primordial de salvaguardar vidas, la seguridad e integridad física de los ciudadanos a quienes se debe su rol como tal, son los rasgos de temperamento en el marco de la personalidad según el resultado del inventario clínico multiaxial de Millón II debe ser dependiente ya que son sujetos que mantiene firme el cumplimento de las normas principios y en el caso de los mismos las normas vigentes en seguridad ciudadana y según el marco teórico de Millon (11) y Eysenck (15) deberán tener un rasgo de temperamento sanguíneo para responder con éxito a la situaciones de riesgo y conflicto.

Los resultados reafirma las conclusiones de la investigación de Matassini (7), titulada, Relación entre inteligencia emocional y clima organizacional en los docentes de una institución educativa publica de La perla Callao, en la que concluyó que si existe una correlación positiva entre ambas variables, encontrándose que a un mayor nivel de inteligencia emocional le corresponde un mejor clima organizacional, también que existe una relación directa y positiva entre la dimensión adaptabilidad de la inteligencia emocional y la variable clima organizacional, los resultados en el personal de seguridad pública las puntuaciones altas de adaptabilidad se relacionan directamente en el afrontamiento de las situaciones difíciles.

Algunas conclusiones son:

El rasgo de personalidad predominante encontrada en el cuerpo de serenazgo y policías municipales, es de personalidad dependiente, seguido por el narcisista, compulsivos y agresivo sádico; afirmando que los rasgos de personalidad guardan relación directa y positiva con la inteligencia emocional.

Los rasgos de personalidad predominantes hallados en el personal de serenazgo y policía municipal en la ciudad de Huancayo al 2015 son dependiente, narcisista y rasgos compulsivos.

Los factores de la inteligencia emocional predominantes encontradas en el personal de serenazgo y policía municipal en la ciudad de Huancayo al 2015 son las habilidades interpersonales, buen autoconcepto, autorealización, adaptabilidad y felicidad.

El grado de significancia en la correlación entre los rasgos de personalidad y la inteligencia emocional en el personal de serenazgo y policía municipal refiere que los rasgos predominantes hallados son los adecuados para brindar una buena seguridad a la ciudadanía que tiene a cargo y que éstas habilidades pueden reforzarse mediante el entrenamiento en 
su inteligencia emocional ya que los rasgos de personalidad son estables y se mantiene en el tiempo.

\section{REFERENCIAS \\ BIBLIOGRÁFICAS}

1. Bisquerra R. Educación emocional y bienestar. Barcelona: Praxis; 2000.

2. Goleman D. Inteligencia emocional. 5 ${ }^{\underline{a}}$ ed. Barcelona: Kairos; 1996.

3. Boeree G. Teorías de la personalidad una selección de los mejores autores de Siglo XX. Santo Domingo: UNIBE; 2005.

4. Bisquerra R. Educación emocional y competencias básicas para la vida. Revista de Investigación Educativa. 2003; 21(1): 7-43.

5. Bisquerra R. Educación para la ciudadanía. El enfoque de la educación emocional. 2a ed. Madrid: Wolters Kluwer; 2008.

6. Cloninger S. Teorías de la personalidad. México: Pearson Educación; 2003.

7. Matassini $\mathrm{H}$. Relación entre inteligencia emocional y clima laboral en los docentes de una Institución Educativa Pública de la Perla, Callao. [Tesis de posgrado]. Lima: Universidad San Ignacio de Loyola; 2012.

8. Blasco J. Educación emocional. 3a ed. Valencia: Generalitat Valenciana; 2002.

9. Hernández R. Metodología de la Investigación. 4a ed. México: McGraw-Hill; 2010.

10. Arias F. El Proyecto de investigación: Introducción a la metodología científica. Caracas: Episteme; 2006.

11. Millon T. Trastornos de la personalidad en la vida moderna. 4a ed. Barcelona: Masson S.A; 2006.

12. Abanto Z, Higueras L, Cueto J. Inventario de cociente emocional de BarOn. Traducción y adaptación para uso experimental en el Perú. Manual técnico. 2a ed. Lima: Grafimag; 2000.

13. Montaño S, Palacios J, Gantiva C. Teorías de la personalidad. Un análisis histórico del concepto y su medición. Psychologia. Avances de la disciplina. 2009; 3(2): 81-107.

14. Contreras F, Barbosa D, Espinosa JC. Personalidad, inteligencia emocional y afectividad en estudiantes universitarios de áreas empresariales. Implicaciones para la formación de líderes. Diversitas. 2010; 6(1): 65-79.

15. Eysenck H. Personalidad y diferencias individuales. Madrid: Pirámide; 1987. 